\title{
Is There a Benefit to Highly Crosslinked Polyethylene in Posterior-stabilized Total Knee Arthroplasty? A Randomized Trial
}

\author{
Paul F. Lachiewicz MD, Elizabeth S. Soileau BSN
}

Published online: 12 March 2015

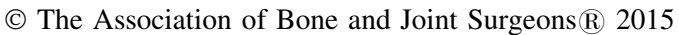

\begin{abstract}
Background Polyethylene wear and osteolysis remain a concern with the use of modular, fixed-bearing total knee arthroplasty (TKA). A variety of highly crosslinked polyethylenes (XLPs) have been introduced to address this problem, but there are few data on the results and complications of this polyethylene in posterior-stabilized knee prostheses.

Questions/purposes In a randomized prospective study design comparing standard polyethylene (SP) tibial inserts with XLP inserts, we asked the following questions: (1) Are there any differences in the clinical results (as measured by the classic Knee Society scoring system) and radiographic results (as measured by an evaluation for radiolucencies and osteolysis) between these groups? (2) What is the frequency of reoperation in these two groups? (3) Are there any specific complications related to XLP liners?
\end{abstract}

The institution of the authors (PFL, ESS) has received funding from Zimmer, Inc (Warsaw, IN, USA).

All ICMJE Conflict of Interest Forms for authors and Clinical Orthopaedics and Related Research ${ }^{\mathbb{R}}$ editors and board members are on file with the publication and can be viewed on request. Clinical Orthopaedics and Related Research ${ }^{\mathbb{R}}$ neither advocates nor endorses the use of any treatment, drug, or device. Readers are encouraged to always seek additional information, including FDAapproval status, of any drug or device prior to clinical use.

Each author certifies that his or her institution approved the human protocol for this investigation, that all investigations were conducted in conformity with ethical principles of research, and that informed consent for participation in the study was obtained.

P. F. Lachiewicz ( $₫)$, E. S. Soileau

Chapel Hill Orthopedics Surgery \& Sports Medicine, 101

Conner Drive, Suite 200, Chapel Hill, NC 27514, USA

e-mail: paul.lachiewicz@gmail.com
Methods These are preliminary data from a single surgeon of a planned interim analysis of a prospective randomized study of one modular posterior-stabilized TKA. One hundred ninety-two patients (236 knees) were randomized to receive a SP compression-molded liner or a XLP (6.5 CGy electron beam-irradiated and remelted) polyethylene liner. There was no difference in the number of knees who were lost or refused followup (14 knees [13\%] with XLP and 21 knees [17\%] with SP). Patients were evaluated clinically using the original Knee Society scores, Lower Extremity Activity Score (LEAS), presence of knee effusion, and by standard radiographs for radiolucent lines and osteolytic lesions. This analysis was performed at a mean followup of 4.5 years (range, 28 years).

Results There were no clinical differences between 99 knees with SP and 94 knees with XLP in original Knee Society total score (SP mean 95, SD 5; XLP mean 94, SD 7 [p $=0.16]$ ); change in total score (SP mean $41,95 \%$ confidence interval [CI], 39-41; XLP mean 43, 95\% CI, 39-48 [p = 0.56]); knee function score (SP mean 64 [SD 25]; XLP mean 64 [SD 24] $\mathrm{p}=0.98$; change in function score (SP mean 22, 95\% CI, 17-27; XLP mean 21, 95\% CI, 17-27 [p = 0.79]); LEAS score (both SP and XLP 9 [SD 2]; $\mathrm{p}=0.88$ ); and change in LEAS (both SP and XLP mean $1,95 \% \mathrm{CI}, 1-2[\mathrm{p}=0.0 .38])$. There were no differences, with the numbers available, in the presence of effusion (two of 94 XLP and five of 99 SP) with 2 years minimum followup. There were no differences in the frequency of radiolucent lines (13 knees with SP and 15 with XLP) or of osteolysis (two knees with SP and none with XLP). There was no difference in frequency of reoperation between the two groups (three infections in 110 knees allocated to the XLP group and five (two infections, one femoral loosening, one instability, one fracture plating) in 
122 knees allocated to the standard group. There were no complications related to the XLP liner.

Conclusions In this interim analysis, with the numbers available, there were no complications, but no advantages, related to the use of this XLP tibial liner. Additional enrollment has continued, and longer followup of these patients will be necessary to determine whether long-term wear characteristics differ between the groups.

Level of Evidence Level I, therapeutic study.

\section{Introduction}

Tibial component polyethylene wear, with resultant particle-induced osteolysis, is one of the causes of late failure, component loosening, and reoperation after TKA [13, 21, 23]. The frequency of periprosthetic osteolysis after TKA has been reported to be $5 \%$ to $20 \%$ at followup of $<5$ to 15 years $[1,6,15,16,21]$. The prevalence and severity of polyethylene wear after TKA are related to multiple factors, including implant design, patient age and activity level, and technical issues. The important implant design factors include tibial component modularity, polyethylene locking mechanism, the method of polyethylene liner fabrication and sterilization, and the "shelf life" of the insert $[1,5,14$, 21]. Technical factors that can accelerate polyethylene wear include coronal and axial malalignment [8].

Highly crosslinked polyethylene (XLP) acetabular liners for THA are now widely accepted as providing decreased linear wear compared with standard polyethylene (SP) in the first decade after implantation [2]. There has been extensive in vitro biomechanical wear testing of several types of modular XLP tibial liners for TKA [6, 8, 18-20, 25]. The clinical use of XLP tibial liners and patellar components was proposed after the early success in THA, but these have not been accepted for routine use [9, 14]. There is concern that some XLP liners show reduced fracture toughness compared with SP $[3,6,25]$ with two reports of three fractures of the tibial posterior-stabilized post of one type of highly XLP at short-term followup [11, $12]$ and two reports of fracture of three XLP patella components $[7,24]$. There is also concern that the size and reactivity of XLP particles may lead to increased periprosthetic osteolysis [6]. We have reported the occurrence of 20 osteolytic lesions in 16 of 132 (12\%) posteriorstabilized knees with SP inserts at a mean followup of 10 years [16]. Osteolysis was more frequent in male patients, in patients with an effusion, and in those with Lower Extremity Activity Score (LEAS) $>10$ [16]. To our knowledge, there are no prospective, randomized studies comparing the clinical and radiographic results and complications of SP and XLP in posterior-stabilized TKA.
We therefore performed a prospective randomized study of both SP and XLP modular tibial liners using the same posterior-stabilized TKA design and asked the following questions: (1) Are there any differences in the clinical results (as measured by the classic Knee Society scoring system) and radiographic results (as measured by an evaluation for radiolucencies and osteolysis) between these groups? (2) What is the frequency of reoperation in these two groups? (3) Are there any specific complications related to XLP liners?

\section{Patients and Methods}

We obtained approval from an institutional review board (Copernicus, Research Triangle Park, NC, USA) for this prospective randomized study with an initial plan to enroll 500 TKAs. This report represents a planned interim analysis, because a review after 5 years of research funding was required. The initial sample size was based on the ability to detect a clinically important difference in postoperative complications and osteolysis between groups. The presumed frequency of osteolysis with SP was based on an earlier study of a different posterior-stabilized knee arthroplasty design [15] and before we had determined the frequency of osteolysis with this second-generation modular posterior-stabilized knee arthroplasty with conventional polyethylene [16]. However, there was no information, to our knowledge, of the frequency of osteolysis with this specific XLP. Based on a frequency of osteolysis of $12 \%$ with SP in that prior study [16], if the frequency of osteolysis with XLP was $1 \%, 70$ knees per group would be needed for a power of $80 \%$. Because there has been an order of magnitude decrease in wear with XLP in THA [2], we considered a frequency of osteolysis of $1 \%$ with XLP in posterior-stabilized TKA to be a reasonable assumption, providing appropriate power for this interim analysis.

Eligibility criteria for study participation included all patients of the surgeon indicated for primary TKA. Patients were excluded if they refused participation, did not comprehend the English language to give informed consent, or if the surgeon believed preoperatively that they would require a primary constrained condylar prosthesis for severe deformity with an incompetent medial collateral ligament.

From December 2005 to July 2012, 249 patients (310 knees) were assessed for eligibility into the study. Informed consent was obtained, by one experienced clinical research nurse (ESS), during the preoperative visit for 192 patients (243 knees). Patients were prospectively randomized by the circulating nurse in the operating room from 2005 to May 2009 and by the research nurse in the clinic (as a result of hospital logistic changes) from July 2009 to the present 


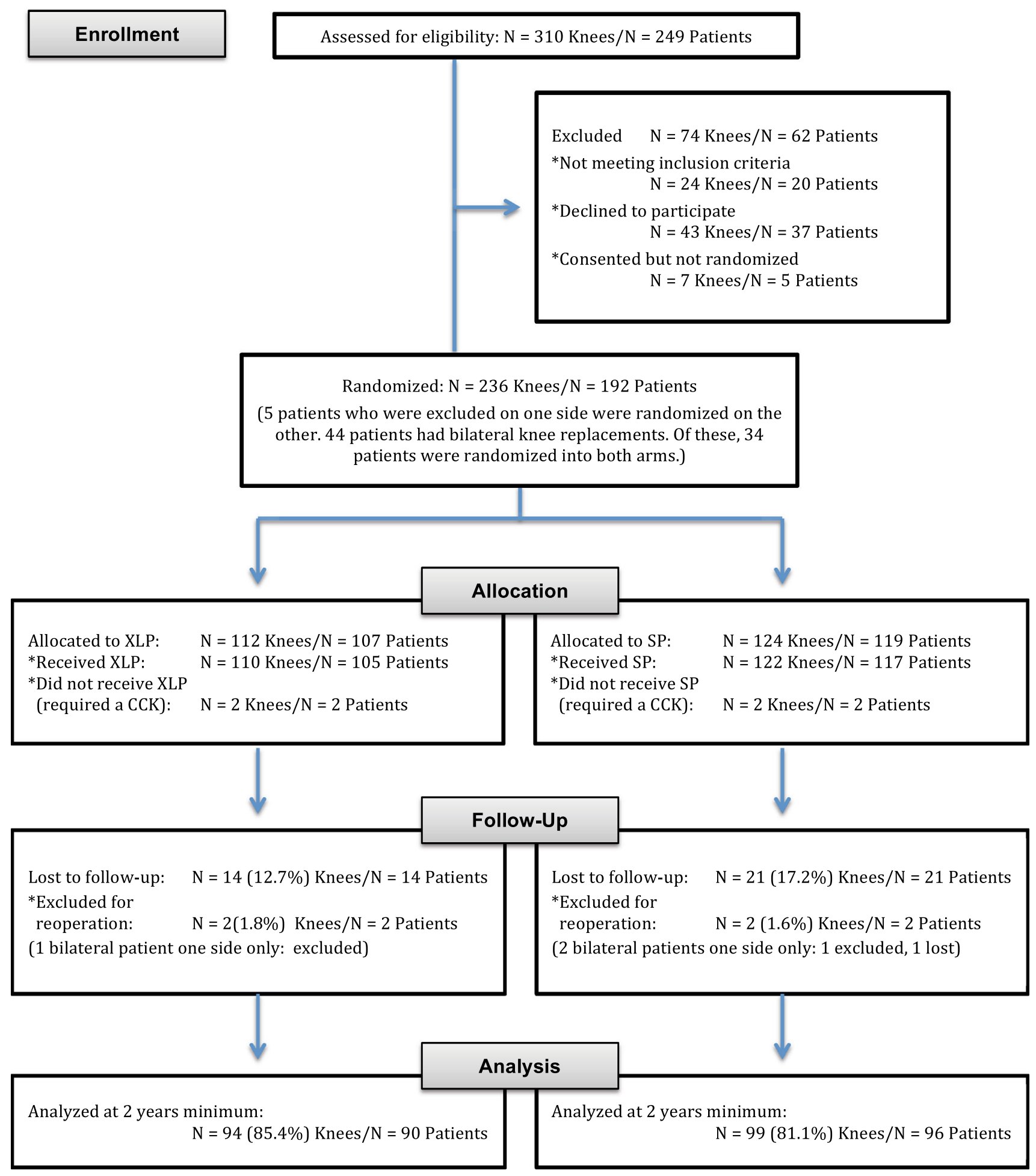

Fig. 1 CONSORT 2010 flow diagram depicts the passage of patients though the randomized controlled trial with details on enrollment, intervention allocation, followup, and analysis. CCK $=$ constrained condylar prosthesis.

using sealed envelopes (both with the surgeon present) to receive either the compression-molded SP (gamma-irradiated in nitrogen) or XLP (electron-beam, 6.5 CGy irradiated in nitrogen, remelted to quench the free radicals; then sterilized by ethylene oxide $\left[\right.$ Prolong ${ }^{\circledR}$ ], both Zimmer,
Inc, Warsaw, IN, USA) (Fig. 1). No liner was used beyond the manufacturer's expiration date.

For the first 4 years of the study, the surgeon performed bilateral one-stage TKAs, and these patients were randomized intraoperatively for the first knee and the second knee received 
Table 1. Comparison of demographic data in all randomized and allocated patients

\begin{tabular}{|c|c|c|c|}
\hline Variable & $\begin{array}{l}\text { Standard } \\
\text { polyethylene } \\
(\mathrm{n}=122 \text { knees })\end{array}$ & $\begin{array}{l}\text { Highly crosslinked } \\
\text { polyethylene } \\
(\mathrm{n}=110 \text { knees })\end{array}$ & $\mathrm{p}$ value \\
\hline Age (mean years; SD) & $70(10)$ & $68(10)$ & 0.18 \\
\hline Gender, male & $37(30 \%)$ & $35(31 \%)$ & 0.79 \\
\hline Body mass index (mean $\mathrm{kg} / \mathrm{m}^{2} ; \mathrm{SD}$ ) & $31(5)$ & $31(6)$ & 0.45 \\
\hline Weight (kg; SD) & $86(15)$ & $87(18)$ & 0.53 \\
\hline Bilateral, one-stage $(\%)$ & $24(19)$ & $24(21)$ & 0.38 \\
\hline Diagnosis of osteoarthritis & $107(88)$ & $98(88)$ & 0.84 \\
\hline \multicolumn{4}{|l|}{ Polyethylene thickness* (mm) } \\
\hline 10 & 61 & 59 & \multirow[t]{2}{*}{0.89} \\
\hline 12 & 45 & 42 & \\
\hline Lost-refused followup, number (\%) & $21(17)$ & $14(13)$ & 0.43 \\
\hline Reoperation knees, number $(\%)$ & $5(4)$ & $3(3)$ & 0.36 \\
\hline
\end{tabular}

* Only the two most frequent thicknesses used are listed.

the other polyethylene liner, serving as their own control. From July 2009 to the present, the surgeon no longer performed bilateral one-stage arthroplasties. These patients were eligible for separate randomization before each arthroplasty.

There were 14 patients (14 knees [13\%]) with XLP and 21 patients (21 knees [17\%]) with SP who were lost or did not return for followup before 2 years $(\mathrm{p}=0.43)$. Of the 14 patients in the XLP group and the 21 patients in the SP group, we reached eight and 11, respectively, by telephone and we were able to determine that they had not had reoperations. Two patients (two knees) in each group who were randomized were excluded intraoperatively when a constrained condylar prosthesis was required for stability. After exclusions for early postoperative reoperations (three infections and one fracture plating), this left 94 knees (90 patients) with XLP and 99 knees (96 patients) with a minimum followup of 2 years. For the 94 knees with XLP, the mean followup was 54 months (range, 24-96 months). For the 99 knees with SP, the mean followup was 53 months (range, 24-96 months).

There were no differences between the groups in patient demographic characteristics (age, gender, weight, body mass index, preoperative diagnosis, number with bilateral onestage and bilateral two-stage procedures), nominal thickness of the tibial polyethylene liner, and followup (Table 1).

Those patients who refused the study received a SP liner and those patients who were excluded and also received a SP liner were routinely evaluated and are included in a "refused study" group (39 knees) to examine the possibility of study bias. Comparing the patients with 39 knees who refused participation in the study and received SP with the randomized group with SP, there was no difference in any of the demographic variables except for weight and body mass index, which were less in the refused group.
All knee surgeries were performed by one surgeon (PFL) through a midline skin incision and a modified Insall medial parapatellar approach using surgical techniques previously described [16]. The patella was routinely resurfaced with a three-peg domed prosthesis fabricated of compression-molded SP (not XLP). Randomization of the patella component was not performed, because we believe wear of this component does not appreciably contribute to osteolysis and this would have added another potential confounding variable. All components were cemented with a two-batch technique using Simplex-P Tobramycin cement (Stryker Howmedica Osteonics, Rutherford, NJ, USA) with a large-bore syringe used for cementing the $7^{\circ}$ fluted, solid titanium alloy tibial component with a dovetail locking mechanism. Postoperatively, all patients ambulated full weightbearing with a walker or crutches on the first postoperative day, had formal physical therapy, and used a continuous passive motion machine while in the hospital. All patients had the same antibiotic prophylaxis (firstgeneration cephalosporin) and thromboembolism prophylaxis (aspirin and mechanical). After discharge, patient received home or outpatient physical therapy until the appropriate goals of ambulation and motion were obtained.

As part of the study, patients were asked to return for routine clinical and radiographic evaluation at 6 months, 1 year, annually until 5 years, and then biannually. The patients were evaluated clinically by one experienced clinical research nurse (ESS) using the classic rating system of the Knee Society (the 1989 original Knee Society scores) for both total knee score and function score [10]. We specifically examined each knee for the presence of a palpable knee effusion (because we previously have found this to be associated with osteolysis) [16] and with an AP drawer test at $90^{\circ}$ flexion to check for an intact tibial post. 
Patients were also assessed pre- and postoperatively at the most recent followup visit using the self-reported LEAS [22].

The knees were evaluated radiographically with standing AP and lateral radiographs and tangential radiographs of the patella in one outpatient location by two technicians specifically trained for these radiographs. The radiographic evaluation system of the Knee Society was used to determine the overall alignment of the knee in the coronal plane, the presence of radiolucent lines in zones adjacent to the cement, and migration of the components using hard copies of radiographs [4]. The alignment of the components in the sagittal plane was not measured. Serial radiographs were reviewed, by both authors together, for changes in the bone that were consistent with osteolysis (defined as wellcircumscribed, circular or oval lytic lesions) and for progression of radiolucent lines $[1,6,15,21]$. There was no analysis concerning intra-/interobserver agreement. Fluoroscopic positioning was not available and not performed in the outpatient clinic location.

Data analysis was performed using standard statistical software (SAS PROC GENMODE, Version 9.3; Cary, NC, USA). In assessing the effectiveness of randomization and the minimum of 2 years of followup results, it was found that $23 \%$ of the subjects had bilateral knees (ie, 44 of 192 subjects) and had both knees enrolled in the study. There was a total of 236 total knee units analyzed in this study. The measurements from these paired knees cannot be considered independent and tend to be positively correlated as a result of common subject-specific characteristics such as age, body mass index, and genetic factors. Therefore, to account for the correlated or clustering effect of these bilateral knees, and to account for the variable time at risk, Generalized Estimating Equations were used as a regression approach on the followup assessments with an independent error structure specified over the variable time at risk with the appropriate distribution specified for the outcome measure (normal for linear, logit for binary). To assess selection bias, a sensitivity analysis was conducted of the patients who refused randomization or were excluded for any reason before randomization, received a SP liner, and had 2-year minimum followup (39 knees); these were compared with the randomized group that received SP. The analysis described was performed, but each knee was considered as an independent observation, because there was a much lower proportion of bilateral knees. Significance was assessed at $\mathrm{p}=0.05$.

\section{Results}

With the numbers available, there were no clinical differences between the 98 knees with SP and 94 knees with XLP in Knee Society total knee score (SP mean 95, SD 5;
XLP mean $94, \mathrm{SD} 7 ; \mathrm{p}=0.16$ ) or change in total knee score (SP mean 41, 95\% confidence interval [CI], 39-41; XLP mean 43, 95\% CI, 39-48; $p=0.56$ ), Knee Society function score (SP mean 64, SD 25; XLP mean 64, SD 24; $\mathrm{p}=0.98$ ), or change in function score (SP mean $22,95 \%$ CI, 17-27; XLP mean 21, 95\% CI, 17-27; $p=0.79$ ), and LEAS score (SP mean 9, SD 2; XLP mean 9, SD 2; $\mathrm{p}=0.88$ ) or change in LEAS score (SP mean $1,95 \% \mathrm{CI}$, $1-2$; XLP mean $1,95 \% \mathrm{CI}, 1-2 ; \mathrm{p}=0.38$ ) (Table 2). There was no difference in the presence of effusion (two of 94 XLP, five of $99 \mathrm{SP} ; \mathrm{p}=0.30$ ). There was no difference in the frequency of radiolucent lines or osteolysis. There were 13 knees with any radiolucent line (three tibia, five femur, five patella) with SP and 15 knees with any radiolucent line (five tibia, seven femur, three patella) with XLP $(p=0.55)$. Osteolysis was present in two knees with $\mathrm{SP}$, both located in zone 1 of the tibia, and there were no lesions seen in knees with XLP $(p=0.49)$. We also compared the knees in the randomized group with SP with the knees in patients who refused the study and received SP. Although there was no difference in Knee Society total score, Knee Society function score, and LEAS scores between the groups, there were more knees with effusion in the "refused group" (seven of 39 compared with five of 98 , $\mathrm{p}=0.04)$. There was one knee in this group with a tibial osteolytic lesion noted at 7 years.

Evaluating the entire randomized group of patients including those requiring a reoperation with less than 2 years followup, there was no difference in frequency of reoperation between the two groups (three, all infections, in 110 knees with XLP; five [two infection, one femoral loosening, one instability, one periprosthetic fracture] in 122 knees with polyethylene, $\mathrm{p}=0.36$ ).

There were no apparent complications related to the XLP tibial liner and the tibial post was intact based on an AP drawer test in all knees examined.

\section{Discussion}

Wear of the modular tibial component polyethylene liner, with resultant particle-induced osteolysis, is one of the causes of synovitis, component loosening, and reoperation in TKA with a frequency of $5 \%$ to $20 \%$ at followups of $<5$ to 15 years $[1,6,15,16,21]$. XLP acetabular liners for THA have provided decreased wear and osteolysis compared with conventional polyethylene in the first decade after implantation [2], but XLP tibial liners have not gained the same acceptance in TKA. There are concerns that some XLP tibial liners show reduced fracture toughness, leading to fracture of the tibial posterior-stabilized post or polyethylene patella component $[3,7,11,12,14,24]$, and that the size and reactivity of XLP particles may actually lead 
Table 2. Outcomes of two randomized cohorts (minimum 2 years followup)

\begin{tabular}{lll}
\hline Variable & $\begin{array}{l}\text { Standard polyethylene } \\
(\mathrm{n}=99)\end{array}$ & $\begin{array}{l}\text { Highly crosslinked } \\
\text { polyethylene }(\mathrm{n}=94)\end{array}$ \\
\hline Followup (mean months; SD) & $53(21)$ & $54(20)$ \\
Knee score, total & & $54(12)$ \\
Preoperative mean (SD) & $54(10)$ & $94(7)$ \\
Followup mean (SD) & $95(5)$ & $43(39-47)$ \\
Change, preoperative to followup, mean (95\% CI) & $41(39-43)$ & $43(21)$ \\
Knee score, function & & $64(24)$ \\
Preoperative mean (SD) & $42(21)$ & $21(17-26)$ \\
Followup mean (SD) & $64(25)$ & 0.74 \\
Change, preoperative to followup, mean (95\% CI) & $22(17-27)$ & $8(2)$ \\
LEAS & & $9(2)$ \\
Preoperative mean (SD) & $8(2)$ & $1(1-2)$ \\
Followup mean (SD) & $9(2)$ & $1(1)$ \\
Change, preoperative to followup, mean $(95 \% \mathrm{CI})$ & $1(1-2)$ & 0 \\
Reoperation for any reason, number $(\%)$ & $3(3)$ & $2(2)$ \\
Any osteolysis, number of knees $(\%)$ & $2(2)$ & $15(16)$ \\
Effusion, number (\%) & $5(5)$ & 0.56 \\
Any radiolucent line, number of knees $(\%)$ & $13(13)$ & 0.98 \\
\hline
\end{tabular}

$* \mathrm{p}$ values are adjusted for nonindependence of correlated measures among patients with bilateral knees; $95 \% \mathrm{CI}=95 \%$ confidence interval; Knee Score = Knee Society (1989) score; LEAS = Lower Extremity Activity Score.

to increased periprosthetic osteolysis [5]. Thus, we performed a prospective randomized study of both SP and XLP modular tibial liners in the same posterior-stabilized TKA design to answer the following questions: (1) Are there any differences in the clinical and radiographic results between a SP and a XLP liner? (2) What is the frequency of reoperation in these two groups? (3) Are there any specific complications related to XLP liners?

This study has several limitations. First, we performed a planned interim analysis of the larger proposed randomized controlled trial for several reasons, including the requirement for a report after 5 years of research funding and the desire on the part of many patients to be apprised of the results to continue followup. Because we had a much lower number of knees with SP, which had osteolysis at this length of followup, the present study is likely underpowered, unless, as previously mentioned, the XLP has a frequency of osteolysis of $1 \%$ or less at 7 to 10 years followup. We have continued to enroll more patients toward our initial study goal of 500 patients and recall all patients for longer clinical and radiographic evaluations; an additional 52 have since been enrolled. Second, after entry into the study, many patients inquired as to which liner they had received and the research nurse informed them. This might have introduced some bias, because patients may have adjusted their activity level thereafter. However, there were no differences in activity level between the groups as measured by either the Knee Society function score or self-reported LEAS score.
There were also no differences between the group randomized with SP and the group that refused the study and received SP. Third, with the initial study design, we had planned for an outside examiner to review the postoperative radiographs, but this later was not feasible as a result of lack of funding and logistic problems. However, the authors were usually not aware of the type of polyethylene liner in the knee when reading the radiographs. Fourth, we did not perform computerized axial tomography or fluoroscopicpositioned radiographs of these knees and the occurrence of osteolysis and radiolucent lines may be underestimated. However, isolated radiolucent lines, unless progressive, have not been considered to correlate with loosening. Fifth, although bilateral knees are not statistically independent endpoints, we included them to have sufficient numbers. As a result of the relatively small number of bilateral TKAs, we do not believe that it affects the findings of this study. Sixth, we implanted an SP patella prosthesis in all knees to eliminate another potential confounding variable. It is unlikely that wear of this nonmetal-backed component contributed to osteolysis or reoperation. Although we lost 35 of the patients $(15 \%)$ to followup before 2 years, there was no differential loss between groups and we are not aware of revisions in these patients. Finally, this study must be considered preliminary, because the followup is likely not sufficient to determine either the true frequency of osteolysis or possible adverse complications related to the SP or XLP liner. 
At a mean followup of 4.5 years (range, 2-8 years), with the numbers available, there were no differences in the clinical or radiographic results between the SP and XLP tibial liners. Randomization appeared effective, because there were no differences in patient demographic data or activity level. However, it is likely that the followup in this and other studies $[9,17]$ is not long enough to definitively answer these questions. There are very few clinical reports of the clinical and radiographic results of XLP tibial liners. One XLP tibial liner (Durasul [9.5 CGy irradiated]; Zimmer, Inc) has been available since 2001. There is one retrospective, nonmatched cohort study that compared 100 knees with SP liners, with 91 months mean followup, to 100 knees with Durasul XLP liners, with 75 months mean followup [9]. There were no clinical results or Knee Society scores reported in this study, but more tibial radiolucent lines were seen with conventional liners (20 of 100 versus two of 100). There were no osteolytic lesions reported. That study has serious methodological limitations, numerous confounding variables, and absence of a measure of activity level $[9,13]$. Another nonrandomized study compared a consecutive series of 113 cruciateretaining TKAs with SP liners with 89 knees with XLP liners (Prolong [6.5 CGy irradiated]; Zimmer, Inc) and reported clinical and radiographic results at 2 years followup [17]. In that series, there were no differences observed between the SP and the XLP liner in terms of Knee Society total knee score, function score, and tibial radiolucent lines. That study reported that no knee had osteolysis. We were not able to show any difference in osteolytic lesions with the numbers available and similarly did not observe clinical differences with longer followup.

In this study, there was no difference in the frequency of reoperation between the SP and XLP groups and no polyethylene issue was implicated in any of the reoperations during this timeframe. In the nonrandomized study of Durasul XLP liners, there was no difference in revisions for an aseptically loose tibial component between the groups (none for the XLP group and three in the SP group) [9]. There were no revisions in either group in the nonrandomized study of SP and Prolong XLP [17].

With the numbers available, there were also no differences in the presence of a knee effusion between the SP and XLP groups. In our previous study of 132 knees (94 patients) with this prosthesis and conventional polyethylene followed for a mean of 10 years (range, 8-14 years), we reported a frequency of $12 \%$ (16 of 132 knees) osteolytic lesions [15]. That study suggested that the presence of palpable knee effusion should alert the surgeon to the possibility of osteolysis in the knee despite few or no symptoms [15]. However, with the numbers available and the relatively short followup to date, we cannot comment on the relative risk between the two groups of osteolysis as a potential cause of reoperation.

At this length of followup, this study has demonstrated no obvious complications or adverse reactions with the use of XLP, posterior-stabilized tibial liners. In particular, we noted no post fractures in the posterior-stabilized inserts. There have been two reports (three knees in three Asian females) with early fracture of a posterior-stabilized tibial post in a highly XLP liner fabricated by sequential irradiation and annealing (X 3; Scorpio; Stryker Howmedica Osteonics) [11, 12]. To our knowledge, there are no reports of fracture of the tibial post of the XLP (Prolong) tibial liner implanted in this study. The XLP is this study was fabricated by electron-beam, 6.5 CGy irradiation, then remelted and sterilized by ethylene oxide. In this study, we used patella components fabricated of SP so as not to introduce a confounding variable and cannot comment on possible complications with the XLP patellar prosthesis.

In conclusion, with the numbers available and with relatively short-term followup, our study suggests that modular tibial posterior-stabilized liners fabricated of this particular XLP provided no clinical and radiographic advantages to SP inserts. Longer followup may show advantages, because radiographic osteolysis and reoperation related to osteolysis are generally not expected at 2 to 5 years. There was no increase in frequency of osteolytic lesions with this specific XLP, as suggested by one laboratory study [6]. There were three knees with SP liners (two in the randomized group and one in the group that refused the study) with osteolytic lesions. Although safety of the XLP cannot be established in a study of this nature [6], no complications directly attributable to the XLP were seen. Based on our previous studies of posterior-stabilized knees, the frequency of effusion resulting from polyethylene wear and osteolytic lesions may increase with longer followup.

At the surgeon's hospital where these knee surgeries were performed, the XLP liner cost was USD 150 more than the SP liner during the period of this study. However, the price differences are quite variable and influenced by geographic location and specific hospital contract with the manufacturer. The manufacturer of this prosthesis supplied this anecdotal information: approximately $60 \%$ of the liners implanted with this knee recently are XLP and $40 \%$ are SP. On the basis of the findings in this interim analysis, we intend to complete enrollment and longer-term followup of this randomized controlled trial. As a result of the absence of major differences at short-term followup between the two liners, it is reasonable for the practicing surgeon to adopt a "wait and see" attitude toward new knee polyethylene products until the longer-term results of this and other studies are completed. 
Acknowledgments We thank Richard Sloane MPH, for the statistical analyses and Stephen Perlman MLS, for assistance with the literature review.

\section{References}

1. Berry DJ. Recognizing and identifying osteolysis around total knee arthroplasty. Instr Course Lect. 2004;440:141-148.

2. Bragdon CR, Doerner M, Martell J, Jarrett B, Palm H, Multicenter Study Group, Malchau H. The 2012 John Charnley Award. Clinical multicenter studies of the wear performance of highly crosslinked remelted polyethylene in THA. Clin Orthop Relat Res. 2013;471:393-402.

3. Cole JC, Lemons JE, Eberhardt AW. Gamma irradiation alters fatigue-crack behavior and fracture toughness in $1900 \mathrm{H}$ and GUR 1050 UHMWPE. J Biomed Mater Res. 2002;63:559-566.

4. Ewald FC. The Knee Society total knee arthroplasty roentgenographic evaluation and scoring system. Clin Orthop Relat Res. 1989;248:9-12.

5. Fehring TK, Murphy JA, Hayes TD, Roberts DW, Pomeroy DL, Griffin WL. Factors influencing wear and osteolysis in press-fit condylar modular total knee replacements. Clin Orthop Relat Res. 2004;428:40-50.

6. Fisher J, McEwen HM, Tipper JL. Wear, debris, and biologic activity of cross-linked polyethylene in the knee: benefits and potential concerns. Clin Orthop Relat Res. 2004;428:114-119.

7. Hambright BA, Watters TS, Kaufman AM, Lachiewicz PF, Bolognesi MP. Fracture of highly cross-linked all-polyethylene patella after total knee arthroplasty. J Knee Surg. 2011;23:237240.

8. Hermida JC, Fischler A, Colwell CW, D'Lima DD. The effect of oxidative aging on the wear performance of highly cross-linked polyethylene knee inserts under conditions of severe malalignment. J Orthop Res. 2008;26:1585-1590.

9. Hodrick JT, Severson EP, McAlister DS, Dahl B, Hoffman AA. Highly cross-linked polyethylene is safe for use in total knee arthroplasty. Clin Orthop Relat Res. 2008;466:2806-2812.

10. Insall JN, Dorr LD, Scott RD. Rationale of the Knee Society clinical rating system. Clin Orthop Relat Res. 1989;248:13-18.

11. Jung KA, Lee SC, Hwang SH, Kim SM. Fracture of a secondgeneration highly cross-linked UHMWPE tibial post in a posterior-stabilized Scorpio knee system. Orthopedics. 2008;31:11371139.
12. Jung KA, Lee SC, Hwang SH, Kim SM. Fractured polyethylene tibial post in a posterior-stabilized knee prosthesis presenting as a floating palpable mass. J Knee Surg. 2009;22:374-376.

13. Lachiewicz MP, Lachiewicz PF. Are the relative indications for revision total knee arthroplasty changing? J Surg Orthop Adv. 2009;18:74-76.

14. Lachiewicz PF, Geyer MR. The use of highly cross-linked polyethylene in total knee arthroplasty. J Am Acad Orthop Surg. 2011;19:143-151.

15. Lachiewicz PF, Soileau ES. Fifteen-year survival and osteolysis associated with a modular posterior stabilized knee replacement. A concise follow-up of a previous report. J Bone Joint Surg Am. 2009;91:1419-1423.

16. Lachiewicz PF, Soileau ES. Fixation, survival and osteolysis with a modern posterior-stabilized total knee arthroplasty. J Arthroplasty. 2014;29:66-70.

17. Minoda Y, Aihara M, Sakawa A, Hawakawa K, Tomita M, Umeda N, Ohzono K. Comparison between highly cross-linked and conventional polyethylene in total knee arthroplasty. Knee. 2009;16:348-351.

18. Muratoglu OK, Bragdon CR, Jasty M, O'Connor DO, Von Knock RS, Harris WH. Knee simulator testing of conventional and crosslinked polyethylene tibial inserts. J Arthroplasty. 2004;428:108-113.

19. Muratoglu OK, Bragdon CR, O'Connor DO, Perinchief RS, Jasty M, Harris WH. Aggressive wear testing of a cross-linked polyethylene in total knee arthroplasty. Clin Orthop Relat Res. 2002;404:89-95.

20. Muratoglu OK, Rubash HE, Bragdon CR, Burroughs BR, Huang A, Harris WH. Simulated normal gait wear testing of a highly crosslinked polyethylene tibial insert. J Arthroplasty. 2007;22:435-444.

21. Naudie DR, Ammeen DJ, Engh GA, Rorabeck CH. Wear and osteolysis around total knee arthroplasty. J Am Acad Orthop Surg. 2007;15:53-64.

22. Saleh KJ, Mulhall KJ, Bershadsky B, Krackow K. Development and validation of a lower extremity activity scale. Use for patients treated with revision total knee arthroplasty. J Bone Joint Surg Am. 2005;87:1985.

23. Sharkey PF, Hozack WJ, Rothman RH, Shastri S, Jacoby SM. Why are total knee arthroplasties failing today. Clin Orthop Relat Res. 2002;404:7-13.

24. Stulberg BN, Wright TM, Stoller AP, Mimnaugh KL, Mason JJ. Bilateral patella component shear failure of highly cross-linked polyethylene components. J Arthroplasty. 2012;27:789-796.

25. Wang A, Yau SS, Essner A, Herrera L, Manley M, Dumbleton J. A highly crosslinked UHMWPE for CR and PS total knee arthroplasties. J Arthroplasty. 2008;23:559-566. 